\title{
Enantioseparation of Three Non-Steroidal Anti-Inflammatory Agents on Chiral Stationary Phase by HPLC
}

\author{
Xuejun Zhang ${ }^{1}$, Zou $\mathrm{Li}^{1,2}$, Baochun Shen ${ }^{1}$, Juanjuan Chen ${ }^{1}$, Xiuzhu $\mathrm{Xu}^{{ }^{*}}$ \\ ${ }^{1}$ Department of Chemistry, Zhejiang University, Hangzhou, China; ${ }^{2}$ Zhejiang Chinese Medical University, Hangzhou, China. \\ Email: *xuxiuzhu@zju.edu.cn
}

Received November $6^{\text {th }}, 2011$; revised December $6^{\text {th }}, 2011$; accepted December $26^{\text {th }}, 2011$

\begin{abstract}
This paper reported the enantioseparation of Naproxen, Ketoprofen and Etodolac on tartardiamide-DMB chiral stationary phase (CHI-DMB) and (R,R)-DNB-DPEDA chiral stationary phase, using hexane as the mobile phase with various modifiers. The influence of the mobile phase composition including the type and concentration of the modifiers in hexane and the structures of the analytes on the chiral separation was studied. The results indicated that the steric structure of the solute, especially the environment of the chiral carbon, was the predominant factor for the chiral recognition. The type, the steric structure and the concentration of the modifiers also influence the retention factor and the resolution. The chiral recognition mechanism of the analytes on CHI-DMB and (R,R)-DNB-DPEDA was also discussed. The hydrogen-bonding interaction played an important role on enantioseparation on CHI-DMB, so did the $\pi-\pi$ interaction. On $(\mathrm{R}, \mathrm{R})$-DNB-DPEDA the $\pi-\pi$ interaction was the key for the solutes retention and enantioseparation, while hydrogen-bonding interaction was not important. Additionally, it has been demonstrated that enantioselectivity and retention was also dependent upon the nature and functional groups on the aromatic ring of solutes and CSP.
\end{abstract}

Keywords: CHI-DMB; (R,R)-DNB-DPEDA; Three Acids; Enantioseparation; Chiral Recognition Mechanism

\section{Introduction}

In recent years, the importance of developing methods for the separation of chiral compounds has been increased in several fields, such as medicine, agrochemicals, asymmetric catalysis, and so on. Chiral recognition plays an essential role in the field of enantioseparation. Chromatographic enantioseparation has become the most practical way of separating enantiomers, and many chiral stationary phases (CSPs) have been developed [1-5]. HPLC separation method based on chiral stationary phases (CSPs) is one of the most powerful and widely used for both analytical and preparative purposes for it enables direct separation of the chiral analytes without preliminary derivation with chiral reagents.

Derivatives of $(R, R)$-tartaric acid, in particular esters and amides, have been used as chiral additives to the mobile phase for the separation of a variety of hydroxy acids and amino acids [6-8]. Mechanistic details of chiral recognition on brush type Pirkle CSPs have been investigated intensely. Its chiral recognition mechanism is relatively better understood and it is possible in many

${ }^{*}$ Corresponding author. cases to predict which analytes will resolve on this CSP [9-12].

Naproxen, ketoprofen, Etodolac belong to an important group of medicines called non-steroidal anti-inflammatory agents and is widely used in the treatment of rheumatic and other inflammatory diseases and for the relief of mild to moderate pain [13]. Naproxen has a chiral centre and the pharmacological activity resides mainly in the $S$-naproxen. Naproxen is sold only as $S$-naproxen [14]. Etodolac is used to relieve the pain, tenderness, inflammation (swelling), and stiffness caused by osteoarthritis and rheumatoid arthritis. Short-acting etodolac also is used to relieve pain from other causes. Etodolac has a chiral centre and used to sold as racemates. But the pharmacological activity resides mainly in the $S$-Etodolac and the $R$-Etodolac almost no pharmacological activity [15]. Therefore, enantiomeric separation and purity assessment is vital in assuring good quality in the pharmaceutical production. A variety of chiral stationary phase (CSP) methods have been proposed for the chiral separation of Naproxen, ketoprofen, such as CHIRALCEL OD, (+) 18-C-6-TA, (R,R)-Whelk-O1, etc. [16-18]. However, there is no literature search about the chiral separation of Naproxen, Etodolac on CHI-DMB CSPs 
and none of them was enantioseparated on (R,R)-DNBDPEDA by HPLC.

In current study the attempt was made to separate the enantiomers of Naproxen, ketoprofen, and Etodolac on CHI-DMB and (R,R)-DNB-DPEDA, and the influence of the mobile phase composition including the type and concentration of the alcoholic modifier in hexane and the structures of the analytes on the chiral separation was investigated. The chiral recognition mechanism between the analytes and CHI-DMB, (R,R)-DNB-DPEDA was also discussed.

\section{Experimental}

\subsection{Regents}

All chemicals used were kindly donated by Zhejiang Agriculture and Farming Administer Office (China) (Figure 1).

\subsection{Apparatus}

Enantioseparations were performed using Waters 2690 Separations Module equipped with a Waters 996 Photodiode Array Detector and Waters Millennium32 System (Waters Co., Milford, MA, USA). Kromasil chiral stationary phase CHI-DMB $(5 \mathrm{~m}, 300 \mathrm{~mm} \times 4.0 \mathrm{~mm}$ i.d.) is based on tartaric acid derivatives which are polymerized, crosslinked, and covalently bound to a functionalized Kromasil silica. (R,R)-DNB-DPEDA (10 m, $250 \mathrm{~mm} \times 4$ $\mathrm{mm}$ i.d.) chiral column were obtained as a gift from Prof. Dr. Kinkel. The structures of the chiral stationary phase are illustrated in Figure 2.

\section{Chromatography}

The mobile phase compositions were $n$-hexane with different percentage of various alcohols, acetic acid (HAC) and tert-butyl methyl ether (TBME). The samples were dissolved in ethanol. All solvents and mobile phase were filtered by $0.45 \mathrm{~m}$ filter membrane and degassed in an ultra-sonic bath before use. The flow rate was $1.0 \mathrm{ml} \cdot \mathrm{min}^{-1}$. The column temperature was $25^{\circ} \mathrm{C}$. UV detection was performed at the maximum absorption wavelength $\left(\lambda_{\max }\right)$ of the solute. The void volume was determined using 1,3, 5tritert-butylbenzene. Separation factors $(\alpha)$ were calculated<smiles>COc1ccc2cc(C(C)(C)C(=O)O)ccc2c1</smiles>

Naproxen

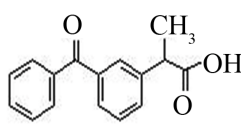

Keroprofen

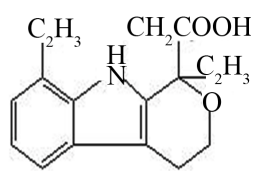

erodolac
Figure 1. The structure of three acides.

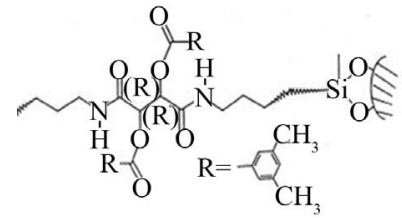

CHI-DMB

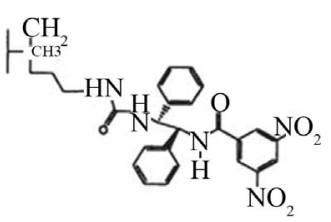

(R,R)-DNB-DPEDA

Figure 2. The structures of chiral stationar phase.

as $\alpha=\mathrm{k}_{2}^{\prime} / \mathrm{k}_{1}^{\prime}$, where $\mathrm{k}_{1}^{\prime}$ and $\mathrm{k}_{2}^{\prime}$ were retention factors for the first and the second eluting enantiomer, respectively. The resolutions $\left(R_{S}\right)$ were calculated as $R_{S}=2\left(t_{2}\right.$ $\left.-t_{1}\right) /\left(w_{1}+w_{2}\right)$, where $w_{1}$ and $w_{2}$ were baseline peak widths for the first and the second eluting enantiomer, respectively. The Kaiser's peak separation index $\left(\mathrm{R}_{\mathrm{p}}\right)$ were calculated as $\mathrm{R}_{\mathrm{p}}=\left(\left(\mathrm{h}_{1}+\mathrm{h}_{2}\right) / 2-\mathrm{h}_{\mathrm{v}}\right) /\left(\left(\mathrm{h}_{1}+\mathrm{h}_{2}\right) / 2\right)$, where $h_{1}$ and $h_{2}$ were the peak heights of the two enantiomers respectively and $h_{v}$ is the valley height between two enantiomer peaks.

\section{Results and Discussion}

\subsection{The Influence of Different Polar Alcohol Modifiers in Mobile Phase on the Enantioseparation of Naproxen, Ketoprofen and Etodolac on CHI-DMB Column}

Using ethanol, n-propanol, iso-propanol, n-butanol as alcoholic modifier respectively, the enantioseparation of Naproxen, Ketoprofen and Etodolac was experimented on CHI-DMB. The chromatographic results are given in Table 1.

It was shown that: 1) The retention factor of naproxen increased as the mobile phase modifier changed from ethanol to iso-propanol and then to n-butanol, should be due to the decrease in solvent polarity and the increase in bulk of alcoholic modifiers. These results indicated that the hydrogen-bonding might be the predominant factor

Table 1. The influence of different polar alcohol modifier in mobile phase on the enantioseparation of three acids on CHIDMB column.

\begin{tabular}{|c|c|c|c|c|c|c|c|c|c|c|c|c|}
\hline \multirow{2}{*}{ solute } & \multicolumn{3}{|c|}{ Hexane-ethanol } & \multicolumn{3}{|c|}{ hexane-n-propanol } & \multicolumn{3}{|c|}{ hexane-iso-propanol } & \multicolumn{3}{|c|}{ hexane-n-butanol } \\
\hline & $\mathrm{K}$ & $\alpha$ & $\mathrm{R}_{\mathrm{S}}$ & $\mathrm{k}_{1}$ & $\alpha$ & $\mathrm{R}_{\mathrm{S}}$ & $\mathrm{k}_{1}$ & $\alpha$ & $\mathrm{R}_{\mathrm{S}}$ & $\mathrm{k}_{1}$ & $\alpha$ & $\mathrm{R}_{\mathrm{S}}$ \\
\hline Naproxen $^{*}$ & 7.31 & 1.09 & 0.96 & 7.23 & 1.1 & 1.13 & 9.2 & 1.1 & 1.05 & 11.5 & 1.11 & 1.08 \\
\hline Ketoprofen ${ }^{*}$ & 10.7 & 1.06 & 0.35 & 10.5 & 1.07 & 0.47 & 12.1 & 1.09 & 0.93 & 13.8 & 1.08 & 0.42 \\
\hline Etodolac ${ }^{* *}$ & 0.83 & 1.31 & 1.85 & 0.95 & 1.35 & 2.11 & 0.92 & 1.36 & 2.33 & 0.95 & 1.37 & 2.19 \\
\hline
\end{tabular}

Stationary phase, CHI-DMB $(5 \mu \mathrm{m}, 300 \mathrm{~mm} \times 4.0 \mathrm{~mm}$ i.d. $)$; mobile phases, ${ }^{*}$ hexane-alcohol, $99: 1,{ }^{* *}$ hexane-alcohol, $99.8: 0.2 ; k_{1}^{\prime}$, the retention factor of the first eluted enantiomer; flow rate, $1.0 \mathrm{ml} \cdot \mathrm{min}^{-1}$; temperature, $25^{\circ} \mathrm{C}$; the dead time was determined using $1,3,5$-tri-tert-butyl benzene; Detection wavelength, Naproxen $(232 \mathrm{~nm})$, ketoprofen $(250 \mathrm{~nm})$, Etodolac $(225 \mathrm{~nm})$. 
between solute and CHI-DMB CSP. In addition, the steric structure and the bulk of the alcoholic modifier were also influence the retention factor. Ketoprofen followed the same trend. But as for Etodolac, the retention factor almost kept constant except ethanol. 2) Etodolac got best enantioseparation among the three non-steroidal anti-inflammatory agents. Compare the structure of the analytes, the chiral carbon of Etodolac directly connects with -O group and the hydrogen-bonding interaction between the -O group of Etodolac and the $\mathrm{N}-\mathrm{H}$ group of the CHI-DMB moiety of CSP played a principle role for the chiral recognition. But the retention of Etodolac was weakly which indicated that stronger interaction didn't mean better resolution and vice versa. 3) Ketoprofen got stronger retention than Naproxen, but obtained worse enantioseparation. As seen in Figure 1, The chiral carbon of Naproxen connects with 6-methyoxy naphthyl group. The phenyl ring of CHI-DMB should be a $\pi$-base because of the two $-\mathrm{CH}_{3}$ groups. And the naphthyl ring with $-\mathrm{OCH}_{3}$ group is a $\pi$-base too, so the $\pi-\pi$ interaction between phenyl ring of CHI-DMB and the the naphthyl ring with $-\mathrm{OCH}_{3}$ group of Naproxen was repulsive. While The chiral carbon of ketoprofen links to metabenzoylphenyl, so the $\pi-\pi$ interaction between CHI-DMB and ketoprofen was attractive. The results suggest that the $\pi-\pi$ interaction was important for solute retention but was not the beneficial for the chiral recognition of three non-steroidal anti-inflammatory agents on CHI-DMB. 4) The $\alpha$ of Naproxen and Ketoprofen was essentially unchanged whenever adding what kind of alcohol. The results indicated that the mobile phase constitution had little affection on enantioselectivity.

\subsection{The Influence of Iso-Propanol Concentration in Hexane on the Chiral Separation on CHI-DMB Chiral Column}

In order to study the influence of the alcohol modifier on the retention factors $\left(\mathrm{k}^{\prime}\right)$, the resolution $\left(\mathrm{R}_{\mathrm{S}}\right)$ and the separation factor $(\alpha)$ for each solutes, the concentration of iso-propanol in hexane was varied. The chromatographic results were given in Table 2.

It was evident that the retention factor and the resolution decreased with increasing of the iso-propanol concentration in mobile phase. As the increase of the alcohol concentration in mobile phase, the eluting ability of mobile phase increased, the strength of the hydrogen bonds between the solutes and stationary phase decreased, then the $\mathrm{k}^{\prime}$ decreased. Under these conditions, the leading interaction between the solutes and the stationary phase was hydrogen bonding interaction. Interestingly, the enantioselectivity of Naproxen and Ketoprofen was essentially unchanged when iso-propanol concentration ranging from $5 \%$ to $1 \%$. But as for Etodolac, the enantioselectivity was changed when iso-propanol concentration ranging from $1 \%$ to $0.2 \%$. This suggested that, at a range
Table 2. The influence of tso-propanol concentration in mobile phase on the enantioseparation of three acids on $\mathrm{CHI}-$ DMB column.

\begin{tabular}{cccccc}
\hline solute & hexane-iso-propanol $(\mathrm{v} / \mathrm{v})$ & $\mathrm{k}_{1}^{\prime}$ & $\mathrm{k}_{2}^{\prime}$ & $\alpha$ & $\mathrm{R}_{\mathrm{S}}$ \\
\hline \multirow{5}{*}{ Naproxen } & $95: 5$ & 1.93 & 2.08 & 1.08 & 0.18 \\
& $96: 4$ & 2.20 & 2.37 & 1.08 & 0.25 \\
& $97: 3$ & 2.91 & 3.17 & 1.09 & 0.42 \\
& $98: 2$ & 3.95 & 4.30 & 1.09 & 0.46 \\
Ketoprofen & $99: 1$ & 9.20 & 10.15 & 1.10 & 1.05 \\
\hline \multirow{5}{*}{ Etodolac } & $95: 5$ & 2.58 & 2.75 & 1.06 & 0.14 \\
& $96: 4$ & 3.01 & 3.22 & 1.07 & 0.21 \\
& $97: 3$ & 3.97 & 4.29 & 1.08 & 0.38 \\
& $98: 2$ & 5.69 & 6.14 & 1.08 & 0.43 \\
& $99: 1$ & 12.1 & 13.2 & 1.09 & 0.93 \\
\hline
\end{tabular}

Mobile phases, hexane-iso-propanol, the other chromatographic conditions were shown in Table 1.

of alcohol modifier concentration, the conformation of the CHI-DMB and the selective adsorption sites were not affected by alcohol modifier concentration. It is also possible that enantiomeric separation was a result of more than one type of interaction. There can be a leading interaction determines the retention of the enantiomers and at least one other type of interaction that is independent of solvent polarity and responsible for enantiomeric selectivity such as steric interactions.

\subsection{The Influence of TBME and HAC Concentration in Hexane on the Chiral Separation on CHI-DMB Chiral Column}

Kromasil chiral stationary phase CHI-DMB is based on tartaric acid derivatives which are polymerized, crosslinked, and covalently bound to a functionalized Kromasil silica. The retention and selectivity are mainly dependent on the hydrogen bonding ability of the analyte, which is regulated by the mobile phase modifiers such as esters, ethers, ketones, and alcohols. In addition to hydrogen bonding there are also $\pi-\pi$ interactions and steric interactions. However, because of the strong hydrogen-donor and acceptor properties of the alcohols, the use of alcohol as an organic phase modifier in the mobile phase inhibited the stereoselective binding between the chiral selector and the analytes, resulting in partial resolution. This is because of competition between the hydroxyl group of the alcohols and the carboxylic group in the analytes occured in forming hydrogen bonds with the respective amide groups on the chiral stationary phase. Replacing alcohols by TBME as an organic mobile phase modifier, these three acids all got better enantiosepara- 
tion. Increasing the amount of TBME reduced the efficiency of resolution. This could be due to an increase of hydrogen bonding between the ether oxygen of TBME and the hydrogen of the amide group in the chiral stationary. Furthermore, the best mobile phase used was hexane and TBME with a small amount of acetic acid. Because the solutes are acidic compounds, the HAC adding can improve peak shape. This may be the addition ensured that the carboxylic group existed in the unionized form $\left(\mathrm{pK}_{\mathrm{a}}\right.$ of acetic acid is 4.74). When the carboxylic group remains in its unionized form, it facilitates forming hydrogen bonding with the amide groups of the chiral selector. Naproxen, ketoprofen and Etodolac were completely resolved since these acides fit the binding sites on the chiral stationary phase, resulting in baseline separation.

The influence of acetic acid concentration in mobile phase on these three acids on CHI-DMB was shown in Table 3.

The data showed the acetic acid adding shortened the retentions dramatically and perfected the band shape. As the increase of acetic acid concentration, solutes got shorter retention and better resolution. It suggested that the competition between the solute and the acetic acid for chiral site on CHI-DMB became stronger, the solute retention went shorter. And the decrease of the TBME concentration and the acetic acid in mobile phase did not have a dramatic effect on the separation factors $(\alpha)$. These results indicated that the polarity alteration of mobile phase did not affect the nature of CSP discrimination.

Table 3. The influence of TBMA and HAC concentration in hexane on the chiral separation on CHI-DMB chiral column.

\begin{tabular}{cccccc}
\hline solute & hexane-iso-propanol(v/v) & $\mathrm{k}_{1}^{\prime}$ & $\mathrm{k}_{2}^{\prime}$ & $\alpha$ & $\mathrm{R}_{\mathrm{S}}$ \\
\hline \multirow{5}{*}{ Naproxen } & $75: 25: 0.1$ & 2.63 & 3.06 & 1.16 & 1.38 \\
& $85: 15: 0.1$ & 4.05 & 4.72 & 1.16 & 1.41 \\
& $95: 5: 0.1$ & 6.89 & 8.01 & 1.16 & 1.64 \\
& $95: 5: 0.2$ & 5.46 & 6.27 & 1.15 & 1.66 \\
Ketoprofen & $95: 5: 0.05$ & 10.7 & 12.6 & 1.17 & 1.92 \\
\hline \multirow{5}{*}{ Etodolac } & $75: 25: 0.1$ & 3.79 & 4.25 & 1.12 & 1.14 \\
& $95: 15: 0.1$ & 6.32 & 7.12 & 1.12 & 1.19 \\
& $95: 5: 0.1$ & 12.5 & 14.1 & 1.13 & 1.36 \\
& $95: 5: 0.2$ & 9.90 & 11.0 & 1.11 & 1.34 \\
& $95: 5: 0.05$ & 19.1 & 21.7 & 1.13 & 1.53 \\
\hline \multirow{5}{*}{} & $95: 25: 0.1$ & 0.54 & 0.72 & 1.33 & 1.62 \\
& $95: 15: 0.1$ & 0.65 & 0.87 & 1.32 & 1.71 \\
& $95: 5: 0.1$ & 0.78 & 1.02 & 1.31 & 1.78 \\
& $95: 5: 0.2$ & 0.73 & 0.93 & 1.27 & 1.57 \\
\hline
\end{tabular}

Mobile phases, hexane-TBMA-HAC, the other chromatographic conditions were shown in Table 1.

\subsection{The Influence of Different Polar Alcohol Modifiers in Mobile Phase on the Enantioseparation of Naproxen, Ketoprofen and Etodolac on (R,R)-DNB-DPEDA Chiral Column}

Naproxen and Etodolac got enantioseparation on $(R, R)$ DNB-DPEDA chiral column but ketoprofen had not been separated. The retention factors $\left(\mathrm{k}^{\prime}\right)$, separation factors $(\alpha)$ and resolutions $\left(R_{s}\right)$ of Naproxen and Etodolac on $(R, R)$ DNB-DPEDA chiral column, with different alcohol modifiers are given in Table 4.

As seen in Table 4, the retention factor of Naproxen was relatively similar on (R,R)-DNB-DPEDA CSP whenever adding what kind of alcoholic modifiers. The isoPropanol was the most favorable modifier and n-Butanol was the most unfavorable modifier for Naproxen. But Etodolac gained best separation using ethanol as alcoholic modifier and obtained worst enantioseparation using iso-Propanol as alcoholic modifier. The results suggested there might not exist hydrogen bonding interaction between solute and CSP or the hydrogen-bonding interaction is not important for the chiral recognition.

\subsection{The Influence of Iso-Propanol Concentration in Hexane on the Chiral Separation on (R,R)-DNB-DPEDA Chiral Column}

The influence of iso-propanol concentration in hexane on the chiral separation was investigated. The chromatographic results are given in Table 5.

As seen in Table 5: 1) The retention factor, enantioselectivity and the resolution decreased with increasing of the iso-propanol concentration in mobile phase. As the decrease of the alcohol concentration in mobile phase, the eluting ability of mobile phase decreased, then the $\mathrm{k}^{\prime}$ increased. The resolution and the separation factor of Naproxen and Etodolac increased as the decrease of the

Table 4. The influence of different polar alcohol modifier in mobile phase on the enantioseparation of Naproxen and Etodolac on (R,R)-DNB-DPEDA column.

\begin{tabular}{lcccccccc}
\hline \multirow{2}{*}{$\begin{array}{c}\text { Alcoholic } \\
\text { modifier }\end{array}$} & \multicolumn{4}{c}{ Naproxen $^{*}$} & \multicolumn{4}{c}{ Etodolac $^{* *}$} \\
\cline { 2 - 9 } & $\mathrm{k}_{1}^{\prime}$ & $\mathrm{k}_{2}^{\prime}$ & $\alpha$ & $\mathrm{R}_{\mathrm{s}}$ & $\mathrm{k}_{1}^{\prime}$ & $\mathrm{k}_{2}^{\prime}$ & $\alpha$ & $\mathrm{R}_{\mathrm{s}}$ \\
\hline Ethanol & 3.22 & 4.13 & 1.28 & 2.50 & 4.28 & 5.56 & 1.30 & 2.42 \\
n-Propanol & 3.18 & 4.05 & 1.27 & 2.48 & 3.82 & 4.88 & 1.28 & 2.28 \\
iso-Propanol & 3.22 & 4.19 & 1.30 & 2.60 & 4.11 & 5.14 & 1.25 & 2.02 \\
n-Butanol & 3.42 & 4.35 & 1.27 & 2.35 & 4.66 & 5.85 & 1.26 & 2.19 \\
tert-Butanol & 3.41 & 4.38 & 1.28 & 2.45 & 4.41 & 5.73 & 1.30 & 2.36 \\
\hline
\end{tabular}

(R,R)-DNB-DPEDA chiral: $(10 \mu \mathrm{m}, 250 \mathrm{~mm} \times 4 \mathrm{~mm}$ i.d.); flow rate: 1.0 $\mathrm{ml} \cdot \mathrm{min}^{-1}$; mobile phase: ${ }^{*}$ hexane-alcohol (95:5); ${ }^{* *}$ hexane-alcohol (98:2); detection wavelength: Naproxen $(232 \mathrm{~nm})$; Etodolac $(225 \mathrm{~nm})$; temperature: $25^{\circ} \mathrm{C}$. 
Table 5. The influence of tso-propanol concentration in mobile phase on the enantioseparation of three acids on $(R$, R)-DNB-DPEDA column.

\begin{tabular}{cccccc}
\hline \multirow{2}{*}{ solute } & $\begin{array}{c}\text { hexane-iso-propanol } \\
(\mathrm{v} / \mathrm{v})\end{array}$ & $\mathrm{k}_{1}^{\prime}$ & $\mathrm{k}_{2}^{\prime}$ & $\alpha$ & $\mathrm{R}_{\mathrm{S}}$ \\
\hline \multirow{5}{*}{ Naproxen } & $80: 20$ & 1.21 & 1.48 & 1.22 & 1.49 \\
& $85: 15$ & 1.41 & 1.77 & 1.25 & 1.80 \\
& $90: 10$ & 1.87 & 2.38 & 1.27 & 2.12 \\
& $95: 5$ & 3.22 & 4.19 & 1.30 & 2.60 \\
\hline \multirow{3}{*}{ Etodolac } & $85: 15$ & 0.87 & & 1 & \\
& $90: 10$ & 1.09 & 1.21 & 1.11 & 0.35 \\
& $95: 5$ & 1.79 & 2.09 & 1.17 & 1.31 \\
& $98: 2$ & 4.11 & 5.14 & 1.25 & 2.02 \\
\hline
\end{tabular}

Mobile phases, hexane-iso-propanol, the other chromatographic conditions were shown in Table 4.

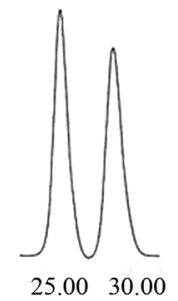

(a)

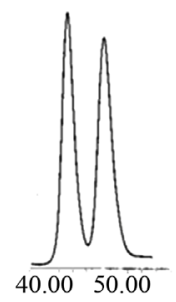

(b)

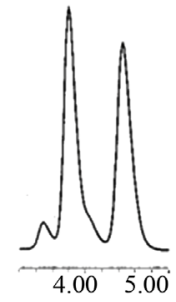

(c)

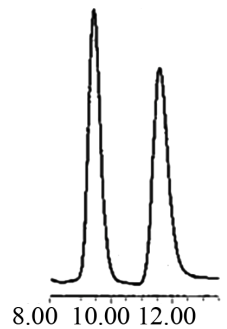

(d)

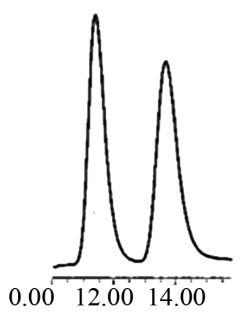

(e)
Chromatographic conditions:

(a) $(\mathrm{S}, \mathrm{S})$-Whelk-O1-CSP; flow-rate, $1.00 \mathrm{ml} \cdot \mathrm{min}^{-1}$; UV detector, $280 \mathrm{~nm}$, mobile phase, $n$-hexane: $i$-propanol $(0.78 \mathrm{~mol} / \mathrm{mL})$ for 1 .

(b) (R,R)-DNB-DPEDA-CSP; flow-rate, $1.00 \mathrm{ml} \cdot \mathrm{min}^{-1}$; UV detector, 302 $\mathrm{nm}$, mobile phase, $n$-hexane: ethnaol $(0.065 \mathrm{~mol} / \mathrm{mL})$ for 2 .

(c) CDMPC-CSP; flow-rate, $0.50 \mathrm{ml} \cdot \mathrm{min}^{-1}$; UV detector, $280 \mathrm{~nm}$, mobile phase, $n$-hexane: $n$-butanol $(0.65 \mathrm{~mol} / \mathrm{mL})$ for 1 .

(d) CDMPC-CSP; flow-rate, $0.50 \mathrm{ml} \cdot \mathrm{min}^{-1}$; UV detector, $302 \mathrm{~nm}$, mobile phase, $n$-hexane: tur-butanol $(0.65 \mathrm{~mol} / \mathrm{mL})$ for 2 .

(e) CTPC-CSP; flow-rate, $0.50 \mathrm{ml} \cdot \mathrm{min}^{-1}$; UV detector, $280 \mathrm{~nm}$, mobile phase, $n$-hexane: tur-butanol $(0.39 \mathrm{~mol} / \mathrm{mL})$ for 1 .

Mobile phases; methanol-HAC-TEA (V/V/V), 100:0.05:0.05, other chromatographic conditions were shown in Table 1.

Figure 3. The enantioseparation of Naproxen (a, d), Ketoprofen (b) and Etodolac (c, e).

alcohol concentration in mobile phase. These results indicated that the polarity alteration of mobile phase affected the nature of (R,R)-DNB-DPEDA CSP discrimination; 2) Naproxen got stronger retention and better resolution than Etodolac. As said before, the chiral carbon of Naproxen connected with 6-methyoxy naphthyl group. The phenyl ring of $(\mathrm{R}, \mathrm{R})-\mathrm{DNB}-\mathrm{DPEDA}$ should be a $\pi$-acid because of the two $-\mathrm{NO}_{3}$ groups. And the naphthyl ring with $-\mathrm{OCH}_{3}$ group is a $\pi$-base, so the $\pi$ - $\pi$ interaction between phenyl ring of $(\mathrm{R}, \mathrm{R})-\mathrm{DNB}-\mathrm{DPEDA}$ and the the naphthyl ring with $-\mathrm{OCH}_{3}$ group of Naproxen was stronger. The results suggest that the $\pi-\pi$ interaction was the key for solute retention and enantioseparation. 3) Ketoprofen had not been separated. The chiral carbon linked to meta-benzoylphenyl, so the $\pi-\pi$ interaction between (R,R)-DNB-DPEDA and ketoprofen was weaker. The results further suggested that the $\pi-\pi$ interaction was the key for solute retention and enantioseparation.

The enantioseparation of Naproxen (a,d), Ketoprofen (b) and Etodolac (c, e) are illustrated in Figure 3.

\section{Conclusion}

In conclusion, Naproxen, ketoprofen and Etodolac got successful enantioseparation on two chiral columns. The chiral recognition mechanism of solutes on each CSP was different. On CHI-DMB, hydrogen-bonding interactions played an important role in chiral recognition. And $\pi-\pi$ interaction was important for solute retention but was not important for the chiral recognition of these acids on CHI-DMB. On (R,R)-DNB-DPEDA-CSP, the $\pi-\pi$ interaction was the key for the solutes retention and enantioseparation, while hydrogen-bonding interaction was not important. Additionally, it has been demonstrated that enantioselectivity and retention was also dependent upon the nature and functional groups on the aromatic ring of solutes and CSP.

\section{Acknowledgements}

We are grateful to Prof. Dr. Kinkel in Georg-Simon-Ohm University of Applied Science (Nürnberg, Germany) for providing (R,R)-DNB-DPEDA chiral column.

\section{REFERENCES}

[1] L. Asnin, F. Gritti, K. Kaczmarski and G. Guiochon, "Features of the Adsorption of Naproxen on the Chiral Stationary Phase (S,S)-Whelk-O1 under Reversed-Phase Conditions," Journal of Chromatography A, Vol. 1217, No. 3, 2010, pp. 264-275.

[2] J. J. Ha, H. J. Choi, J. S. Jin, E. D. Jeong and M. H. Hyun, "Liquid Chromatographic Resolution of Proton Pump Inhibitors Including Omeprazole on a Ligand Exchange Chiral Stationary Phase," Journal of Chromatography A, Vol. 1217, No. 41, 2010, pp. 6436-6441. doi:10.1016/j.chroma.2010.08.041

[3] K. Si-Ahmed, F. Tazerouti, A. Y. Badjah-Hadj-Ahmed, Z. Aturki and G. D'Orazio, "Optical Isomer Separation of Flavanones and Flavanone Glycosides by Nano-Liquid Chromatography Using a Phenyl-Carbamate-Propyl- $\beta$ Cyclodextrin Chiral Stationary Phase," Journal of Chromatography A, Vol. 1217, No. 7, 2010, pp. 1175-1182. doi:10.1016/j.chroma.2009.07.053 
[4] L. L. Zhou, B. Mao and Z. H. Ge, "Comparative Study of Immobilized $\alpha 1$ Acid Glycoprotein and Ovomucoid Protein Stationary Phases for the Enantiomeric Separation of Pharmaceutical Compounds," Journal of Pharmaceutical and Biomedical Analysis, Vol. 46, No. 5, 2008, pp. 898-906. doi:10.1016/j.jpba.2007.07.008

[5] L. M. Peng, S. Jayapalan, B. Chankvetadze and T. Farkas, "Reversed-Phase Chiral HPLC and LC/MS Analysis with tris(Chloromethylphenylcarbamate) Derivatives of Cellulose and Amylose as Chiral Stationary Phases," Journal of Chromatography A, Vol. 1217, No. 44, 2010, pp. 6942-6955. doi:10.1016/j.chroma.2010.08.075

[6] J. Oxelbark and P. Gidlund, "Investigation of a Tartaric Acid-Based Linear Polyamide and Dimer as Chiral Selectors in Liquid Chromatography," Chirality, Vol. 17, No. 2, 2005, pp. 79-84. doi:10.1002/chir.20106

[7] H. Y. Aboul-Enein, "Chiral Resolution of Some Piperidine 2,6-dione Drugs by High Performance Liquid Chromatography on Kromasil@ CHI-DMB Column," Archiv der Pharmazie, Vol. 337, No. 8, 2004, pp. 453- 456. doi:10.1002/ardp.200300857

[8] Y. F. Liu, C. P. Zhai, M. H. Zhou, J. G. Zheng, Q. Xiao, Q. Z. Li, D. Li, D. H. Xiao and D. L. Yue, "Direct Resolution of Diniconazole Optical Isomers on Chiralcel Acylamide Column," Journal of Instrumental Analysis, Vol. 28, No. 10, 2009.

[9] B. Kosjek and G. Uray, "Immobilization of Difunctional Building Blocks on Hydroxysuccinimide Activated Silica: Versatile in Situ Preparation of Chiral Stationary Phases," Chirality, Vol. 13, No. 10, 2001, pp. 657-667. doi:10.1002/chir.10020

[10] D. M. Forjan, D. Kontrec and V. Vinković, "Performance of Brush-Type HPLC chiral Stationary Phases with Tertiary Amide in the Connecting Tether," Chirality, Vol. 18,
No. 10, 2006, pp. 857-869. doi:10.1002/chir.20329

[11] B. C. Shen, X. Z. Xu, X. J. Zhang and J. J. Chen, "Enantioseparation and Chiral Recognition Mechanism of Two Novel Organic Phosphonate Derivatives on Chiral Stationary Phases," Analytical Letters, Vol. 38, 2005, pp. 1317-1330.

[12] W.-J. Wei, H.-W. Deng, W. Chen, Z.-W. Bai and S.-R. Li, "Preparation and Enantioseparation of a Mixed Selector Chiral Stationary Phase Derived from Benzoylated Tartaric Acid and 1,2-Diphenylethylenediamine," Chirality, Vol. 22, No. 6, 2010, pp. 604-611.

[13] A. Campiglio, "Determination of Naproxen with Chemiluminescence Detection," Analyst, Vol. 123, 1998, pp. 1571-1574. doi:10.1039/a802013b

[14] F. Lelievre and P. Gareil, "Chiral Separations of Underivatized Arylpropionic Acids by Capillary Zone Electrophoresis with Various Cyclodextrins Acidity and Inclusion Constant Determinations," Journal of Chromatography $A$, Vol. 735, No. 1-2, 1996, pp. 311-320. doi:10.1016/0021-9673(95)00745-8

[15] J. M. Shi and D. Li, Chinese Journal of Clinical Pharmacy, Vol. 9, 2000, pp. 321-324.

[16] D. M. Chen, Q. Fu, N. Li, S. X. Zhang and Q. Q. Zhang, Chinese Journal of Chemistry, Vol. 35, No. 1, 2007, pp. 75-78.

[17] K. W. Tang, P. L. Zhang, C. Y. Pan and H. J. Li, "Modeling Multiple Chemical Equilibrium for Reactive Extraction of Naproxen Enantiomers with HP- $\beta-\mathrm{CD}$ as Hydrophilic Selector," Science China Chemistry, Vol. 54, No. 7, 2011, pp. 1130-1137.

[18] Q. Tang, X. Y. Chen and H. Song, Chinese Journal of Chemistry, Vol. 35, No. 2, 2007, pp. 240-243. 